\title{
Haematological toxicity in cancer cervix patients treated with concurrent chemoradiation by conventional technique- correlation with bone marrow radiation dose
}

\author{
Upadhyay P. ${ }^{1}$, Kumar $P^{2 *}{ }^{2}$, Kumar $P^{3}{ }^{3}$, Garg A. ${ }^{4}$, Chauhan A.K. ${ }^{5}$ \\ DOI: https://doi.org/10.17511/ijmrr.2020.i03.03
}

1 Upadhyay P., Junior Resident, Department of Radiation Oncology, Shri Ram Murti Smarak Institute of Medical Sciences, Bareilly, Uttar Pradesh, India.

${ }^{2 *}$ Kumar P., Assistant Professor, Department of Radiation Oncology, Shri Ram Murti Smarak Institute of Medical Sciences, Bareilly, Uttar Pradesh, India.

${ }^{3}$ Kumar P., Professor, Department of Radiation Oncology, Shri Ram Murti Smarak Institute of Medical Sciences, Bareilly, Uttar Pradesh, India.

${ }^{4}$ Garg A., Assistant Professor, Department of Radiation Oncology, Shri Ram Murti Smarak Institute of Medical Sciences, Bareilly, Uttar Pradesh, India.

${ }^{5}$ Chauhan A.K., Professor, Department of Radiation Oncology, Shri Ram Murti Smarak Institute of Medical Sciences, Bareilly, Uttar Pradesh, India.

Introduction: The standard of care for treatment of cancer cervix is concurrent chemoradiation followed by brachytherapy in the majority of cases. Conventional radiotherapy with chemotherapy causes haematological toxicities which may be related to radiation to pelvic bone marrow. Material and Methods: Retrospective data of cancer patients treated in the institute in the year 2019 was retrieved. Haematological toxicities were analyzed in terms of CTCAE criteria. Mean dose to bone marrow was calculated after the delineation in the CT scan. Results: The data of 20 patients were retrieved. Anaemia Grade, I and Grade II-IV was seen in $65 \%$ and $35 \%$ respectively. Leukopenia Grade I and Grade II-IV were seen in $85 \%$ and $15 \%$ respectively and Lymphopenia Grade I and Grade II-Iv were seen in 55\% and $45 \%$ respectively. Conclusion: Conventional radiotherapy can safely be practice for patients with cancer cervix with acceptable haematological toxicities.

Keywords: Cancer cervix, Haematological toxicities, Mean dose bone marrow

Corresponding Author

Kumar P., Assistant Professor, Department of Radiation Oncology, Shri Ram Murti Smarak Institute of Medical Sciences, Bareilly, Uttar Pradesh, India. Email: pawan.mehrotra12@rediffmail.com
How to Cite this Article

Upadhyay P, Kumar P, Kumar P, Garg A, Chauhan AK. Haematological toxicity in cancer cervix patients treated with concurrent chemoradiation by conventional technique- correlation with bone marrow radiation dose. Int J Med Res Rev. 2020;8(3):234-239.

Available From

https://ijmrr.medresearch.in/index.php/ijmrr/article/ view/1199
To Browse

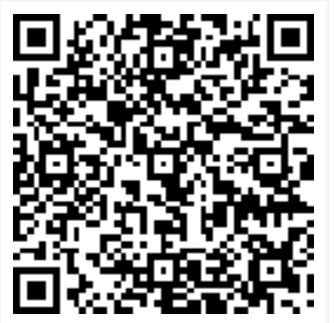

Manuscript Received 2020-05-20

Conflict of Interest No

Review Round 1
2020-05-30
Funding
Nil

2020 by Upadhyay P., Kumar P., Kumar P., Garg A., Chauhan A.K. and Published by Siddharth Health Research and Social Welfare Society. This is an Open Access article licensed under a Creative Commons Attribution 4.0 International License https://creativecommons.org/licenses/by/4.0/ unported [CC BY 4.0]. 


\section{Introduction}

Concurrent chemoradiation followed by brachytherapy is the standard of care for cervical cancer $[1,2]$. The role of this combined therapy is to maximise tumour cell death with the radiosensitizing effects of chemotherapy. Traditionally conventional radiotherapy fields $(4$ field box technique) were based on bony anatomy, which has resulted in good loco-regional controls, but the geographic miss of the clinical target volume may often result in an increased risk of failures and the large volumes treated resulted in an increase in gastrointestinal (GI), genitourinary (GU) and haematological toxicity (HT) [3]. The patients are planned radiotherapy by conventional technique to the whole pelvis which includes the tumour, pelvic lymph nodes along with organs at risk like the rectum, urinary bladder, bone marrow (BM) and small intestine. Bone marrow is a radiosensitive tissue and there is a strong link between the dose and the volume irradiated and the risk of haematological toxicity. The decline in bone marrow hematopoietic cells is associated with an increased level of adipocytes and a chronic inhibition of hematopoiesis [4], as confirmed by experimental studies [5]. In adults' pelvic bone is the primary site of haematopoiesis. It is estimated that $>50 \%$ of proliferating BM is located in the pelvic region, including lumbar spine [6]. The irradiation to the whole pelvis may lead to suppression of bone marrow resulting in the decrease of haematological parameters. This may lead to anaemia, neutropenia and thrombocytopenia. The toxicity may further be enhanced by the chemotherapy drugs commonly used for the treatment of cancer cervix during radiotherapy. The newer radiotherapy techniques like intensity-modulated radiotherapy (IMRT) are emerging to decrease the dose to the BM. However, the ability of IMRT to reduce the PBM dose, particularly low-dose radiation, compared with conventional forward planning techniques have not been fully investigated [7-9]. This decrease in dose to the bone marrow, theoretically seems may decrease the haematological toxicities, but the clinical advantage is still controversial. IMRT is a costly radiotherapy technique and not available in most of the centres of the country. The present study aims to study the haematological toxicities caused due to chemoradiation in patients of cancer cervix treated with conventional radiotherapy technique and try to find out if there is any correlation between radiotherapy dose to the pelvic
Bone marrow and haematological toxicities.

\section{Material and Methods}

Study Setting: Department of Radiation Oncology, Shri Ram Murti Institute of Medical Sciences

Duration of study: January 2019 to December 2019

Type of Study: Observational study.

Sampling methods: Retrospective data of all the patients of cancer cervix treated in the year 2019 were selected

Study tool: Correlation between the mean dose of bone marrow and haematological toxicity was evaluated.

Inclusion criteria: Biopsy proven cervical carcinoma, 18-65 years age and KPS $\geq 70$

Exclusion criteria: Previous history radiation or chemotherapy, synchronous malignancy or metastatic disease

Data collection procedure: Retrospective data of all the patients of cancer cervix treated with conventional radiotherapy technique along with concurrent chemotherapy in the year 2019 were selected who had received 50 Gy in 25 fractions at 2 Gy per fractions over 5weeks followed by 3 fractions of ICRT of $7 \mathrm{~Gy}$ each along with weekly Cisplatin (35mg/m2) concurrently.

Radiation Planning: All the patients had similar radiotherapy planning technique. They were immobilized with the thermoplastic cast and a planning computed tomography of the pelvis (CT RTP) with $3 \mathrm{~mm}$ slice thickness was taken. The radiotherapy was planned by four-field box technique (anterior-posterior, posterior-anterior and two laterals) using standard bony landmarks.

Bone Marrow Contouring: Bone marrow was contoured in all these patients as described by Mell et al[10]. The external contour of all bones within pelvis was contoured on planning $\mathrm{CT}$ scan depicting BM. The external contour was chosen, rather thanthe low- density regions within bones, to ensure reproducibility and to minimizes dependence of the contours on CT windowing and levelling. Whole pelvis bone marrow included the following bones-

- Ilium-including the iliac crests extending to the superior border of the femoral head

- Lower pelvis- consisting of the pubis, ischia, 
Acetabula, and proximal femora extending from the superior border of the femoral head to the inferior of the ischial tuberosities

- Lumbosacral spine-extending superiorly from the appearance of the iliac bone and inferiorly to include the entire sacrum

Retrospective data were retrieved which included details of various haematological parametershaemoglobin $(\mathrm{Hb})$, total leukocyte count (TLC), lymphocyte count (LC), neutrophil count (NC) and platelets.

Any scoring procedure: Mean dose (Dmean) of BM was calculated. Hematologic toxicity was graded according to CTCAE v4.03.

\section{Surgical procedure: None}

Ethical consideration and permission: Not required

Statistical analysis: Correlation between Dmean of BM and haematological toxicity was seen using paired t-test and $\mathrm{p}$-value less than 0.05 was considered significant.

\section{Results}

Twenty patients of cancer cervix were treated with concurrent chemoradiation in the year 2019. The haematological parameters and mean doses of BM are shown in Table 1.

Table-1: Haematological parameters and mean bone marrow dose of each patient $(n=20)$.

\begin{tabular}{|c|c|c|c|c|c|c|}
\hline \multirow[t]{2}{*}{ S.No. } & \multicolumn{5}{|c|}{ Haematological parameters } & \multirow[t]{2}{*}{ Mean Bone Marrow dose (Gy) } \\
\hline & $\mathrm{Hb}(\mathrm{g} / \mathrm{dL})$ & TLC (cells/mm3) & LC (\%) & NC (\%) & Platelets (lacs/mm3) & \\
\hline 1 & 8.6 & 3300 & 6 & 83 & 1.78 & 34.79 \\
\hline 2 & 11.1 & 3600 & 13 & 71 & 2.2 & 27.51 \\
\hline 3 & 10.9 & 4200 & 9 & 56 & 1.9 & 35.75 \\
\hline 4 & 8 & 2900 & 7 & 81 & 1.45 & 40.75 \\
\hline 5 & 11.5 & 5000 & 10 & 72 & 1.4 & 34.42 \\
\hline 6 & 9.3 & 6800 & 6 & 70 & 1.4 & 34.70 \\
\hline 7 & 10 & 6500 & 6 & 59 & 1.56 & 38.44 \\
\hline 8 & 10.9 & 4200 & 9 & 76 & 1.9 & 38.50 \\
\hline 9 & 10 & 4400 & 7 & 72 & 2.6 & 36.81 \\
\hline 10 & 7.7 & 2600 & 11 & 60 & 2.65 & 33.57 \\
\hline 11 & 9.9 & 3700 & 8 & 76 & 1.22 & 34.16 \\
\hline 12 & 9 & 4900 & 7 & 65 & 2.82 & 36.55 \\
\hline 13 & 6.6 & 5800 & 7 & 75 & 3 & 38.25 \\
\hline 14 & 8 & 8700 & 5 & 86 & 4.6 & 33.30 \\
\hline 15 & 9.7 & 3900 & 10 & 63 & 2.98 & 33.80 \\
\hline 16 & 10.8 & 3300 & 8 & 74 & 1.9 & 41.70 \\
\hline 17 & 9.1 & 2600 & 7 & 82 & 1.2 & 34.52 \\
\hline 18 & 8.1 & 8000 & 10 & 71 & 2.2 & 38.89 \\
\hline 19 & 10.4 & 3700 & 9 & 81 & 1.1 & 34.56 \\
\hline 20 & 10.1 & 6800 & 11 & 85 & 2.1 & 33.49 \\
\hline
\end{tabular}

Haematological toxicity was stratified into mild grading (Grade I) and moderate to severe grading (Grade II to IV) and correlated with mean dose to bone marrow (Table 2).

Table-2: Correlation of haematological toxicities with the mean dose of bone marrow.

\begin{tabular}{|l|l|l|l|l|}
\hline Haematological Toxicity & Grading & n (\%) & Dmean (Gy) & P-value \\
\hline \multirow{2}{*}{ Anaemia } & Grade I & $13(65)$ & 35.41 & 0.5 \\
\cline { 2 - 4 } & Grade II-IV & $7(35)$ & 36.3 & \\
\hline
\end{tabular}

\begin{tabular}{|l|l|l|l|l|}
\hline Leukopenia & Grade I & $17(85)$ & 35.62 & 0.8 \\
\cline { 2 - 4 } & Grade II-IV & $3(15)$ & 36.28 & \\
\hline Lymphopenia & Grade I & $11(55)$ & 35.53 & \multirow{2}{*}{0.38} \\
\cline { 2 - 4 } & Grade II-IV & $9(45)$ & 35.95 & \\
\hline
\end{tabular}

There was no Grade II-IV toxicity of neutropenia and thrombocytopenia.

\section{Discussion}

Bone marrow is a radiosensitive tissue and the irradiation may cause its suppression leading to 
Various haematological toxicities like anaemia, leukopenia and thrombocytopenia. Albuquerque et al [2] treated patients with conventional radiotherapy and all patients had some grade of hematologic toxicity. Moderate to severe toxicity (Grade 2 or more) was observed in $67.5 \%$ of patients. In the present study also, all patients developed some grade of anaemia, leukopeniaand lymphopenia. Around $30 \%$ of patients showed haematological toxicity in terms of neutropenia and thrombocytopenia. On the contrary, the majority of patients in the present study had grade 1 toxicity and only a few patients had toxicity more than grade 2. The RTOG 0418 [11] phase II clinical trial showed that the volume of bone marrow receiving $40 \mathrm{~Gy}$ or more had higher rates of grade 2 toxicity. In the present study, the mean dose of bone marrow was less than 40Gy and this may be the possible reason why our majority of patients had grade 1 haematological toxicity. In the RTOG 0418 phase II clinical radiotherapy technique IMRT was used whereas the present study utilized conventional radiotherapy. It clearly shows that complex and advance techniques though may show the theoretical advantage, may not always give better clinical outcomes. In a study by Gandhi AK et al [12] the haematological toxicity was compared between whole pelvis conventional radiotherapy (WP-CRT) and IMRT (WP-IMRT). In WP-CRT arm $54.5 \%$ patients had more than grade 2 and $18.2 \%$ had more than grade 3 haematological toxicity, whereas $72.7 \%$ had more than grade 2 and more than $13.6 \%$ had more than grade 3 toxicity in WPIMRT arm. The difference in haematological toxicities was statistically non-significant. It is important to note that most of the centres in our country do not have higher modalities of radiotherapy treatment and routinely practice conventional radiotherapy. In this scenario, the study by Gandhi AK et al concluding that both WPCRT and WP-IMRT had comparable clinical outcomes supports the practice of standard conventional radiotherapy techniques and eliminates the feeling of the patient being devoid of advanced techniques. Ashitha EA et al [13] in their study found that IMRT reduces the volume of bone marrow getting irradiated to higher doses and decreasing the severity of acute haematological toxicities. Patients with IMRT had fewer more than grade 2 toxicities $28 \%$ vs $72 \%$ compared to 3DCRT $(p=.03)$. The results of this study are contrary to haematological toxicity by the conventional technique in the present study. The present study would like to suggest that if the clinical outcomes in terms of tumour control
Are comparable in both conventional and advanced techniques, there is no reason why the standard practice of conventional radiotherapy cannot be continued. The newer techniques like IMRT may cause an unnecessary financial burden on the patient and may not be always a good choice for patients who belong to a developing country. Recently, there have been several reports on the applications of BM-sparing IMRT, which was thought to effectively reduce the irradiated volume of BM compared with conventional techniques. In patients with cancer cervix, Brixey et al [7] showed that IMRT was associated with both lower HT and lower BM dose compared with patients treated with conventional 4-field box techniques, indicating that IMRT could be used to reduce HT. Iliac, lumbar, and sacral BM irradiation was reduced with IMRT, even though the plans were not optimized to spare the BM. Subsequent studies demonstrated that IMRT and intensity-modulated arc therapy plans could be optimized to improve IBM sparing $[9,14]$. In postoperative cervical cancer patients, Chen et al. [15] have previously shown that, without entering PBM as a planning constraint, IMRT reduced PBM irradiation compared with four-field box techniques. Others have shown that IMRT plans can be optimized to reduce BM irradiation compared with conventional techniques with extended-field and whole abdomen RT $[16,17]$. The addition of concurrent chemotherapy in the treatment of cervical cancer has led to improved tumour control and survival [18-21], generally at the expense of increased toxicity, particularly HT. A legitimate concern is whether HT isenough of a problem in cervical cancer to warrant the application of the highly technical treatment, particularly when the predicted advantages compared with a simple APPA plan are not clear. The reported acute HT with whole pelvic RT and concurrent chemotherapy in cervical cancer trials has been variable, with acute Grade 3 or greater HT about 35\% in some studies [22] but much lower in others [23]. In our experience, and others [10.15,24], acute Grade 3 or greater $\mathrm{HT}$ has been relatively low with concurrent weekly cisplatin and RT, although Grade 2 or greater HT has approached to about $45 \%$. Therefore, the potential benefits of BMS-IMRT might be better realized by combining it with more aggressive chemotherapy. This study may support the use of high-end technology, but at the same time, it also incorporates the higher cost of treatment. Our present study has acceptable mild grades of haematological toxicities in the majority of patients. The treatment by conventional radiotherapy is 
Cheaper, affordable and available in most of the centres across the country. IMRT may be offered to a selected class of patients where cost is not a barrier.

\section{Limitations}

Small sample size and using the entire bone as a proxy for BM constitutes another limitation of the study.

\section{Conclusion}

Conventional radiotherapy can be safely practised for the patients of cancer cervix with acceptable haematological toxicities. The newer techniques like IMRT which are costly and not available in most of the parts of the country may not show a clinical advantage in terms of decreasing the haematological toxicities.

\section{What does the study add to the existing knowledge}

In the era of advancement in radiotherapy technology, but the limitation of availability of such techniques develops a feeling of compromising the treatment in the minds of treating physician and the patient. This study suggests that the conventional technique of radiotherapy in patients of cancer cervix is no way inferior in terms of treatmentrelated haematological toxicities when compared to advanced techniques.

\section{Author's Contribution}

Dr. Prachi Upadhyay: Collection data and manuscript review

Dr. Pavan Kumar: Drafting methodology, verification of data and review of the manuscript

Dr. Piyush Kumar: Drafting the methodology, drafting and review of the manuscript

Dr. Ayush Garg: Collection data and manuscript review

Dr. Arvind Kumar Chauhan: Manuscript review

\section{Reference}

01. Thomas GM. Improved treatment for cervical cancer-concurrent chemotherapy and radiotherapy. N Engl J Med. 1999;340(15)11982000.

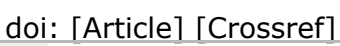

02. Albuquerque K, Giangreco D, Morrison C. Radiation related predictors of hematologic toxicity after concurrent chemoradiation for cervical cancer and implication for bone marrow sparing pelvic IMRT. Int Radiat Oncol Biol Phys. $2011 ; 79(4) 1043-47$. doi: [Article] [Crossref]

03. Roeske JC, Lujan A, Rotmensch J, Waggoner SE, Yamada D, Mundt AJ. Intensity-modulatedwhole pelvic radiation therapy in patients with gynecologic malignancies. Int J Radiat Oncol Biol Phys. 2000;48(5)1613-1621. doi: [Article] [Crossref]

04. Green DE, Rubin CT. Consequences of irradiation on bone and marrow phenotypes, and its relation to disruption of hematopoietic precursors. Bone. 2014;63;87-94.

doi: [Article] [Crossref]

05. McGuire SM, Bhatia SK, Sun W, Jacobson GM, Menda $Y$, Ponto $L L$, et al. Using [18F] fluorothymidine imaged with positron emission tomography to quantify and reduce hematologic toxicity due to chemoradiation therapy for pelvic cancer patients. Int J Radiat Oncol Biol Phys. 2016;96(1)228-239.

doi: [Article] [Crossref]

06. Hayman JA, Callahan JW, Herschtal A, Everitt S, Binns DS, Hicks RJ, et al. Distribution of proliferatingbone marrow in adult cancer patients determined using FLT-PET imaging. Int J Radiat Oncol Biol Phys. 2011;79(3)847-852. doi: [Article] [Crossref]

07. Brixey CJ, Roeske JC, Lujan A, et al. Impact of intensity-modulated radiotherapy on acute hematologic toxicity in women with gynecologic malignancies. Int J Radiat Oncol Biol Phys. 2002;54(5)1388-1396. doi: [Article] [Crossref]

08. Mundt AJ, Lujan A, Rotmensch J, Yamada SD, Rotmensch J, Mundt AJ. Intensity-modulated whole pelvic radiotherapy in women with gynecologic malignancies. Int J Radiat Oncol Biol Phys. 2002;54(5)1330-1337. doi: [Article] [Crossref]

09. Lujan A, Mundt AJ, Yamada D, Rotmensch J, Roeske JC. Intensity-modulated radiotherapy as a means of reducing dose to bone marrow in gynecologic patients receiving whole pelvic radiotherapy. Int J Radiat Oncol Biol Phys. 2003;57(2)516-521.

doi: [Article] [Crossref] 
10. Mell LK, Kochanski JD, Roeske JC, Haslam JJ, Mehta N, Yamada DS, et al. Dosimetric predictors of acutehematologic toxicity in cervical cancer patients treated with concurrentcisplatin and intensity-modulated pelvic radiotherapy. Int J Radiat Oncol Biol Phys. 2006;66(5)1356-1365.

doi: [Article] [Crossref]

11. Klopp AH, Moughan J, Portelance L, Miller BE, Salehpour MR, Hildebrandt E, et al. Hematologic toxicity in RTOG 0418- a phase 2 study of postoperative IMRT for gynecologic cancer. Int J Radiat Oncol Biol Phys. 2013;86(1)83-90. doi: [Article] [Crossref]

12. Gandhi AK, Sharma DN, Rath GK, Julka PK, Subramani V, Sharma S, et al. Early Clinical Outcomes and Toxicity of Intensity Modulated Versus Conventional Pelvic Radiation Therapyfor Locally Advanced Cervix Carcinoma- A Prospective Randomized Study. Int J Radiat Oncol Biol Phys. 2013;87(3)542-548.

doi: [Article] [Crossref]

13. Ashitha EA, Bindu G, Ajayakumar T, Janish K, Jayaraman MB. Association between acute hematological toxicities and bone marrow dosimetric parameters in cervical cancer patients undergoing concurrent chemoradiationa comparison between threedimensional conformal radiotherapy and intensity modulated radiotherapy. Int $\mathrm{J}$ Contemp Med Res. 2018;5(11)1-5. doi: [Article] [Crossref]

14. Wong $E$, D'Souza DP, Chen JZ, Lock $M$, Rodrigues $G$, Coad $T$, et al. Intensity-modulated arctherapy for treatment of high-risk endometrial malignancies. Int J Radiat Oncol Biol Phys. 2005;61(3)830-841. doi: [Article] [Crossref]

15. Chen MF, Tseng CJ, Tseng CC, Kuo YC, Yu CY, Chen WC. Clinical outcome in post hysterectomy cervical cancer patients treated with concurrent cisplatin and intensity-modulated pelvic radiotherapy- Comparisonwith conventional radiotherapy. Int J Radiat Oncol Biol Phys. 2007;67(5)1438-1444.

doi: [Article] [Crossref]

16. Hong L, Alektiar K, Chui C, LoSasso T, Hunt M, Spirou $S$, et al. IMRT of large fields- Whole abdomen irradiation. Int $\mathrm{J}$ Radiat Oncol Biol Phys. 2002;54(1)278-279.

doi: [Article] [Crossref]
17. Ahmed RS, Kim RY, Duan J, Meleth S, Santos JF De L, Fiveash JB. IMRT dose escalation forpositive para-aortic lymph nodes in patients with locally advanced cervical cancer while reducing dose to bone marrowand other organs at risk. Int $\mathrm{J}$ Radiat Oncol Biol Phys. 2004;60(2)505-512.

doi: [Article] [Crossref]

18. Keys HM, Bundy BN, Stehman FB, Muderspach LI, Chafe WE, Suggs CL 3rd, et al. Cisplatin, radiation and adjuvant hysterectomy compared with radiation and adjuvant hysterectomy for bulky stage IB cervical carcinoma. N Engl J Med. 1999;340(15)1154-1161. doi: [Article] [Crossref]

19. Morris M, Eifel PJ, Lu J, Grigsby PW, Levenback $C$, Stevens $R E$, et al. Pelvic radiation with concurrent chemotherapy compared with pelvic and para-aortic radiation for high-risk cervical cancer. N Engl J Med. 1999;340(15)1137-1143. doi: [Article] [Crossref]

20. Rose PG, Bundy $B N$, Watkins $E B$, Thigpen JT,Deppe G, Maiman MA, et al. Concurrent cisplatin based radiotherapy and chemotherapy for locally advanced cervicalcancer. $N$ Engl J Med. 1999;341(9)1144-1153.

doi: [Article] [Crossref]

21. Green JA, Kirwan JM, Tierney JF, P Symonds, L Fresco, $M$ Collingwood $M$, et al. Survival and recurrence after concomitant chemotherapy and radiotherapy for cancer ofthe uterine cervix- $A$ systematic review and meta-analysis. Lancet. $2001 ; 358(9284) 781-786$.

doi: [Article] [Crossref]

22. Peters WA III, Liu PY, Barrett RJ II, Stock RJ, Monk BJ, Berek JS, et al. Concurrent chemotherapy and pelvic radiation therapy compared with pelvic radiation therapy alone as adjuvant therapy after radical surgery in highrisk early-stage cancer of the cervix. J Clin Oncol. 2000;18(8)1606-1613.

doi: [Article] [Crossref]

23. Pearcey R, Brundage $M$, Drouin P,Jeffrey J, Johnston $D$, Lukka $H$, et al. Phase III trial comparing radiocal radiotherapy with and without cisplatin chemotherapy in patients with advanced squamous cell cancer of thecervix. J Clin Oncol. 2002;20(4):966-972.

doi: [Article] [Crossref] 
24. Torres MA, Jhingran A, Thames HD Jr, Levenback CF, Bodurka DC, Ramondetta LM, et al. Comparison oftreatment tolerance and outcomes in patients with cervical can certreated with concurrent chemoradiotherapy in a prospective randomized trial or with standard treatment. Int J Radiat Oncol Biol Phys. 2008;70(1)118-125.

doi: [Article] [Crossref] 